\title{
The role of radiotherapy in urinary bladder cancer: current status
}

Gustavo Nader Marta, Samir Abdallah Hanna, Rafael Gadia, Sebastião Francisco Miranda Correa, Joao Luis Fernandes da Silva, Heloisa de Andrade Carvalho

Department of Radiation Oncology - Oncology Center, Hospital Sirio-Libanes (GNM, SAH, RG, SFMC, JLFS, HAC), Sao Paulo, Brazil; Department of Radiation Oncology - Oncology Center Instituto do Cancer do Estado de Sao Paulo (RG), Sao Paulo, Brazil; Department of Radiation Oncology - Oncology Center, Hospital da Real e Benemerita Sociedade Portuguesa de Beneficencia (SFMC), Sao Paulo, Brazil and Radiation Oncology, Department of Radiology, Faculty of Medicine, University of Sao Paulo, (HAC), Sao Paulo, Brazil

\section{ABSTRACT}

The role of radiotherapy (RT) in the treatment of urinary bladder cancer has undergone several modifications along the last decades. In the beginning, definitive RT was used as treatment in an attempt to preserve the urinary bladder; however, the results were poor compared to those of radical surgery. Recently, many protocols have been developed supporting the use of multi-modality therapy, and the concept of organ preservation began to be reconsidered. Although phase III randomized clinical studies comparing radical cystectomy with bladder preservation therapies do not exist, the conservative treatment may present low toxicity and high indexes of complete response for selected patients. The aim of this study was to review the literature on the subject in order to situate RT in the current treatment of urinary bladder cancer.

\section{ARTICLE INFO}

\section{Key words:}

radiotherapy; urinary bladder; neoplasms; review

Int Braz J Urol. 2012; 38: 144-54

Submitted for publication:

April 11, 2011

Accepted after revision:

August 05, 2011

\section{INTRODUCTION}

An estimated 386,300 new cases and 150,200 deaths from bladder cancer occurred in 2008 worldwide (1).

The TNM (2) staging system is the most important independent prognostic variable in invasive urinary bladder cancer. This system is used for urothelial carcinoma, squamous cell carcinoma, undifferentiated carcinoma and urinary bladder adenocarcinoma. The spectrum of urinary cancer includes non-muscle invasive (superficial) tumors, muscle invasive tumors and metastatic disease. Each one of these tumors presents a specific clinical behavior, prognostic and treatment. Regardless of the tumoral stage, the standard treatment for urinary bladder cancer is surgery. From endoscopic resections to radical cystectomies, surgery still offers the best locoregional control (3). However, radical cystectomy may be associated with a decrease in the patients' quality of life, especially the functional quality of the neo-bladder, even taking into account the advances of the reconstruction techniques used 
for this organ (4-6). Occurrences such as urinary incontinency and erectile dysfunction (in cases in which radical prostatectomy was performed during the surgery) are not rare and can be the tiebreakers for choosing a conservative option of organ preservation.

Historically, radical radiotherapy (RT) with curative intent was offered only to patients with severe clinical comorbidities, or in an advanced stage of the disease, or older age, at the moment of the diagnostic. With the advances of the therapeutic modalities, combined multidisciplinary treatment is, at present, the one that offers the best possibilities of cure with preservation of the bladder (7).

Up to this date, there are no phase III randomized studies that compare radical surgery versus more conservative multimodality approaches. Only informations from retrospective studies or non randomized prospective series are available. Besides, RT also plays an important role in symptoms palliation in curable or incurable cases and presents different indications in the treatment of urinary bladder cancer.

This study presents a literature review, in order to situate RT in the current curative treatment of urinary bladder cancer.

\section{Radiotherapy for superficial cancer}

Information on the use of RT in the treatment of superficial urinary bladder cancer dates from before the success obtained with endoscopic treatments. Nowadays, there is no support in the literature that justifies its routine use in this group of patients (8-10).

In most reports, $\mathrm{RT}$ is used in patients with progressive disease or in recurrences, after many transurethral resections of bladder tumors (TURB) combined or not with intravesical therapy.

Despite of the relatively high rate of complete responses in stages Tis and $\mathrm{Ta}$, it is known that approximately 50\% of the patients will present recurrence in the first year after RT; in five years, this rate will be 90\% (11).

There is no evidence that RT offers a higher probability of urinary bladder preservation than other treatments (TURB and intravesical therapy). However, Weiss et al. (12) suggested that TURB followed by radiochemotherapy could be an alternative treatment for high-risk tumors (T1, G3). In view of the satisfactory results obtained with TURB and BCG, the use of RT in this context is rarely justified and therefore, should be considered only in an individual basis.

\section{Radiotherapy for Invasive Cancer}

\section{Preoperative Radiotherapy}

Preoperative RT emerged in an attempt to minimize the possible dissemination of the disease during surgery, as well as to eradicate microscopic tumor focuses that might had been situated beyond the resection margins.

At the beginning, many retrospective studies confirmed a real benefit in the use of preoperative RT compared to cystectomy alone (13-15). A review published by the University of Florida (16) in the 80's with stage T3 patients concluded that preoperative RT would give a 5 -year survival benefit of 5\% (15\% versus 20\%) when compared to surgery alone.

Conflicting results were found in studies that compared preoperative RT followed by immediate radical surgery versus radical treatment with RT and salvage surgery at recurrence (17-21). It is important to note that the RT scheme used was different in each of these studies. And, most of the times, a small number of patients were included which limited the statistical power of these analyses. Except for the MD Anderson experience (Miller) (21) that showed statistically significant benefit favoring bimodal therapy, the other studies did not find any difference in terms of patient's survival in the evaluated groups. However, the study of Miller also must be interpreted with caution considering the small number of patients analyzed and the fact that only patients with large T3 tumors were considered.

Moreover, a meta-analysis involving five randomized studies concluded that the available data from those clinical studies do not support the routine use of preoperative RT (22).

Therefore, although it has been considered an option in the past, preoperative RT is not recommended in the treatment of invasive urinary bladder cancer. 


\section{Postoperative Radiotherapy}

After radical cystectomy, RT is rarely administered in the postoperative setting of patients with urinary bladder cancer. This is mainly due to the presence of high rates of side effects related to irradiation in the postsurgical period, after the abdominal manipulation and consequent fixation of both large and small bowel to the pelvis, as the neo-bladder itself. Complication rates vary around $20 \%$. Nevertheless, the pelvic control of the disease is in general satisfactory with surgery only (13),

Positive margins or lymph node involvement are adverse risk factors for recurrence and patient survival $(23,24)$.

Postoperative RT should be considered only in individualized situations such as recurrences of pelvic disease.

\section{Definitive Radiotherapy}

Until the development of modern surgical techniques for the performance of cystectomy, RT was widely used as a radical and exclusive treatment modality. Nowadays, RT alone should be used only in patients who present a high risk of complications during surgery or with advanced disease.

RT effectiveness in the treatment of urinary bladder cancer was repeatedly demonstrated in retrospective studies in Europe and Canada. However, these studies, most of the time, demonstrated lower local control and shorter patient survival when compared to cystectomy.

The results of a Scottish retrospective study revealed that $45.9 \%$ out of 963 patients, treated with RT alone, presented local tumoral regression (25). An English retrospective study showed $40 \%$ five-year survival with $41 \%$ of local control in 182 patients with T2 and T3 urinary bladder cancer treated with RT (26).

A Norwegian retrospective series (27) focused on patient survival after radical treatment of transitional cell carcinoma of the bladder. Forty five patients underwent cystectomy, and ninety patients underwent RT. After surgery, the ten-year overall survival for superficial and muscle-invasive tumors was, respectively, 67\% and $26 \%$ and for patients treated with RT, 26\% and $5 \%$, respectively.
Fossa et al. (28) studied patients with T2 to T4 bladder cancer in whom total cystectomy could not be performed due to medical contraindications and were treated with curative RT alone. Five-year survival was 22\%.

A study performed at Princess Margareth Hospital (29) in Toronto reported results after a long follow-up period of patients with invasive bladder cancer (T1 to $\mathrm{T} 4$ ) treated with radical RT. Of 340 patients evaluated, 247 received RT alone. Complete response rate was $63.5 \%$ for the whole group. Overall and cause-specific survival and local control rate in ten years was 19\%. In 131 patients with disease limited to the bladder wall (T2NOM0), cause-specific survival and local control rate in ten years were 68\% and 60\% (p = 0.02 ) with the absence of carcinoma in situ and $47 \%$ and $28 \%(\mathrm{p}=0.03)$ with the presence of carcinoma in situ, respectively. In the multivariate analysis, younger age, lower T stage, and absence of carcinoma in situ were associated to a significant increase in local control and survival ( $p \leq$ 0.01 ). The study concluded that RT-based treatment is a good alternative to radical cystectomy, regarding local control and survival and may be used in selected patients.

There is a sub-group of patients who may benefit from RT alone: T2 tumors, patients with no urethral obstruction, complete transurethral resection, solitary tumor and absence of T4 disease.

A study conducted by Shipley et al. at the Massachusetts General Hospital (30) revised the results of 55 patients treated with RT alone in order to identify factors associated with tumor radioresponsiveness and patient cure. Sixty seven percent of the patients presented T2 or T3 clinical stage and 33\% were T4. Overall 5-year survival was $28 \%$ for the whole group. When comparing T2/T3 with T4 patients, survival was $45 \%$ versus $9 \%$, respectively $(\mathrm{p}=0.009)$. Within $\mathrm{T} 2 / \mathrm{T} 3$ group the most important prognostic factor was the histological finding of tumor on the papillary surface of the bladder, with 63\% local control rate and 62\% 5-year survival versus 20\% and $0 \%$ in the presence of solid or bulky tumors. Other important prognostic factors in five-year survival in this group were the extent of complete transurethral resection (54\% complete versus 17\% incom- 
plete, $\mathrm{p}=0.009$ ) and urethral obstruction in the intravenous pyelogram (47\% without obstruction versus $14 \%$ with obstruction, $p=0.01$ ). The study concluded that RT may be used in the treatment of muscle-invasive tumors, with a better probability of success in patients with less advanced clinical stages, in the presence of tumors on the papillary surface, in the absence of urethral obstruction, and with the possibility of complete transurethral resection.

Similar findings were published by Mameghan et al. (31) in which bad prognostic factors for vesical recurrence were tumor multiplicity, the presence of urethral obstruction and larger tumor size.

Recently, a German series (32) reported the results of 75 patients with localized muscleinvasive bladder carcinoma $(\mathrm{T} 2, \mathrm{n}=34$; $\mathrm{T} 3, \mathrm{n}=$ 32 ; $\mathrm{T} 4, \mathrm{n}=9$ ). Patients were considered not suitable for radical surgery due to advanced age, comorbidity or inoperability. Definitive RT was delivered with tridimensional conformal technique without concomitant chemotherapy. Complete response was obtained in $2 / 3$ of the patients and the 3-year overall survival rate was 56.9\%. Although the short follow-up, they concluded that $\mathrm{RT}$ is an effective treatment option in terms of local control and survival even in elderly patients with locally advanced bladder cancer not suitable for cystectomy.

\section{Multimodal therapy}

The rational of a multidisciplinary approach of invasive bladder cancer is based on the promising results of combined therapeutic modalities when compared to radical surgery.

TURB is used to reduce the tumor volume to be irradiated. Moreover, it is known that transitional cell carcinoma of the bladder is highly chemoresponsible. Chemotherapy attempts to eradicate local and systemic disease and to increase RT effect on locoregional control. This multimodality approach was pioneered by Housset et al. (33).

The National Cancer Institute of Canada Clinical Trials Group (34) in a prospective randomized study showed the benefits of adding chemotherapy to RT in patients with muscle invasive bladder cancer. Patients with T2 to T4 tumors were randomized to receive or not concomitant chemotherapy with RT. A lowest percentage of pelvic recurrence was observed in the arm that received the combined therapy (59\% versus 40\% $p=0.038$ ). Overall survival after three years was higher in the group that received cisplatin (47\% versus 33\%), however, with no statistical significance $(p=0.34)$.

Recently, a phase III study (BC 2001 CRUK/01/04) (35) demonstrated that radiochemotherapy increases the locoregional control of tumors invading the muscular layer, when compared to RT alone, with preservation of the vesical function and without increase in acute or late toxicity. Patients were randomized to receive 5-fluorouracil (5-FU) with mytomicin C, concomitant to RT and a 2-year locoregional control rate of $67 \%$ was achieved, compared to $54 \%$ for patients submitted to RT only.

Until now, there are no level I evidence studies that compared radiochemotherapy with radical cystectomy. Data obtained from prospective and retrospective series point out the real benefits of the combination. The largest experiences are from the groups of the Massachusetts General Hospital, USA, of the University of Erlangen, Germany, and of the University of Paris, France. Combined treatment protocols (TURB followed by radiochemotherapy) come from these institutions.

The German study, conducted by Rödel et al. (36), analyzed the combined treatment with bladder preservation and tried to identify factors that could predict treatment response, risk of recurrence and survival. They evaluated 415 patients (89 high risk T1; $326 \mathrm{~T} 2$ to $\mathrm{T} 4$ ) who were treated with RT (126) or radiochemotherapy (289) after TURB of the tumor. Six weeks after treatment, the response was evaluated by re-staging TURB. In case of a complete response, the patients were kept under observation in regular intervals. In case of tumor persistence or invasive tumor recurrence, salvage cystectomy was recommended. Seventy two percent of the patients had complete response with maintenance of local control and $64 \%$ of the patients did not present recurrence, after 10 years of follow-up. Distant metastases were diagnosed in 98 patients with a 35\% rate 
in 10 years. Ten-year disease-free survival was $42 \%$ and more than $80 \%$ of the surviving patients had their urinary bladder preserved. Radiochemotherapy was more effective than RT alone regarding local control and survival. The initial stage of the tumor and a complete TURB were the most important predictive factors of both local control and survival.

Weiss et al. (12) included 112 patients with muscle invasive urinary bladder cancer or high risk T1 (grade 3, associated Tis, multifocal, diameter $>5 \mathrm{~cm}$ ). The protocol consisted of TURB followed by cisplatin and 5-FU administered from days 1 to 5 and 29 to 33 of RT. Treatment response was evaluated by re-staging TURB 4 to 6 weeks after radiochemotherapy. In case of residual invasive tumor or recurrence, salvage cystectomy was recommended. Complete response was observed in $88 \%$ of the patients, and $72 \%$ did not present local or distant recurrence. Overall and cause-specific survival rates for all patients were $74 \%$ and $85 \%$ in 5 years, respectively. Of all surviving patients, $82 \%$ remained with their own bladder and of these, 79\% were satisfied with their urinary condition.

Patients with high risk T1 tumors (G3, associated Tis, multifocal, or recurrent) should be evaluated with caution. In many of them, TURB followed by intravesical therapy (BCG or chemotherapy, for example) is used. However, a considerable percentage of these patients (15 to 40\%) present local recurrence, jeopardizing the possibilities of initial cure (37). This is the reason why some authors indicate radical cystectomy as the first line standard treatment for these patients. As a consequence of these uncertainties, many patients are undertreated (TURB followed by intravesical therapy) or overtreated (radical cystectomy as initial treatment). Moreover, many patients submitted to staging with TURB may be understaged, presenting more invasive tumors in up to one third of the cases $(38,39)$. Considering this, RT would play a role in the treatment of tumors invading deeper layers and in eventually involved lymph nodes. The results presented in Weiss's study (12) seem to be superior when compared to TURB studies followed by intravesical BCG $(40,41)$ and are relatively equivalent to radi- cal cystectomy (42). This suggests that TURB followed by radiochemotherapy may be an adequate treatment for patients with high risk T1 stage.

A more recent study by Caffo et al. (43) supports these findings. They studied 26 patients who underwent transurethral tumor resection followed by a radical dose of external radiotherapy with concomitant cisplatin and weekly gemcitabine therapy. With a median follow-up of 74 months, 5-year overall survival rate was $70.1 \%$, with 5-year disease-specific survival and bladder-intact survival rates of 78.9\% and 73.8\%, respectively.

So, in general, we can consider that RT alone is inferior to the combined scheme with chemotherapy. Also, it is important to note that the complete pathological response rates observed in re-evaluation cystectomy or at the end of the combined treatment varies from 50\% to $90 \%$, being consistent in approximately $70 \%$ of the patients. Five-year overall survival is approximately $60 \%$ and about at least half of the patients maintains a functional urinary bladder after 5 years of follow-up. However, much of the evidence of the multimodality approach including endoscopic resection and radiotherapy combined with chemotherapy is retrospective and involves treating locally advanced poor-risk patients (44). Prospective evaluation of these treatments for truly localized (T1/2) bladder cancer should be encouraged.

\section{Induction chemotherapy followed by radiother- apy/radiochemotherapy}

For a long time, many institutions established two cycles of induction chemotherapy before radiochemotherapy for the treatment of tumors in an advanced stage (45). However, more recently, many authors have questioned the success of urinary bladder preservation based on the treatment with induction chemotherapy $(46,47)$.

Arias et al. (48) in a prospective study included 50 patients in good clinical conditions who had operable urinary bladder invasive cancer (T2 to T4). The treatment protocol was as follows: 1) TURB; 2) two cycles of chemotherapy (methotrexate, vinblastine, adriamycin and cisplatin - MVAC); 3) 45 Gy RT with concomitant chemotherapy (cisplatin); 4) cytoscopic evaluation: in the presence of a complete response, RT was complet- 
ed until 65 Gy; if the response was incomplete, cystectomy was performed. Sixty eight percent of the patients had a complete response. Five-year local control and overall survival were $48 \%$ and $47 \%$, respectively. For patients who presented a complete response, 65\% and 70\%, respectively.

The role of induction chemotherapy was tested in a phase III study conducted by the Radiation Therapy Oncology Group (RTOG) (49). The objective of this study was to test the efficacy of neoadjuvant methotrexate, cisplatin and vinblastine (CMV) in patients with muscle-invasive urinary bladder cancer treated with an organ preservation approach. One hundred and twenty three patients (clinical stage T2 to T4aNXM0) were randomized to receive pelvic irradiation (39.6 Gy) with concomitant cisplatin with (arm 1, 61 patients) or without (arm 2, 62 patients) two cycles of neoadjuvant CMV. Patients that presented complete response received additional RT (25.2 Gy) with one more dose of cisplatin. Patients whose responses were not complete were submitted to cystectomy. Seventy four percent of the patients completed the protocol (67\% arm 1 and 81\% arm 2). Five-year overall survival was 49\% (48\% arm 1 and 49\% arm 2). Thirty five percent of the patients presented evidence of distant metastases in 5 years (33\% arm 1; 39\% arm 2). Survival rate in 5 years with functioning bladder was 38\% (36\% arm 1; 40\% arm 2). These differences were not statistically significant.

Additionally, Perdonà et al. (50) reported a promising result with 2 cycles of neoadjuvant chemotherapy followed by radiotherapy $(\mathrm{n}=43$ patients) or radiochemotherapy ( $\mathrm{n}=78$ patients). Complete response was observed in 102 out of 119 evaluable patients. With a median follow-up of 66 months, the 5-year tumor-specific, overall, and bladder-intact survival rates were $73.5 \%$, $67.7 \%$, and $51.2 \%$, respectively.

The larger randomized study of neoadjuvant chemotherapy using the CMV regimen ( 3 cycles), followed either by surgery or RT or both, was conducted by the Medical Research Council / European Organization for Research and Treatment of Cancer (MRC/EORTC) with 976 patients (51). At first, a non-significant increase of overall survival in the group that received neoadjuvant therapy was observed (HR $=0.85 ; 95 \%$ CI: 0.71 - $1.02 ; p=0.075)$. The results of this study were recently updated and the survival advantage of neoadjuvant CMV became statistically significant with $16 \%$ reduction in the risk of death (hazard ratio, $0.84 ; 95 \% \mathrm{CI}, 0.72$ to $0.99 ; \mathrm{p}=0.037$, corresponding to an increase in 10-year survival from $30 \%$ to $36 \%$ ) after CMV (52).

Concluding, for deeply invasive bladder cancer, induction chemotherapy with CMV followed by definitive local therapy presents a clinically relevant survival benefit and may be considered as first-line adjunctive treatment and should be viewed as the state of the art, as compared with cystectomy or radiotherapy alone (52).

Alterations in Radiotherapy Fractionation

Usually, RT is fractionated in 1.8 to 2 Gy per day, 5 days a week. A total dose of 45 to 50 Gy is delivered to the pelvis and 55 to 70 Gy to the bladder tumor bed, achieving favorable rates of local control (53).

A Dutch review (54) emphasized the importance of the treatment dose in which $10 \mathrm{~Gy}$ increments in the final dose of RT yielded an increase of about 50\% in local control rate in three years.

Some groups have used RT schemes with altered fractionation (hyperfractionated or accelerated fractionation) in an attempt to improve the results of irradiation. Hyperfractionation consists in increasing the number of fractions per day, with a lower dose per fraction, but with an increase in the total daily dose, ending the therapy in a period similar to conventional RT, with an absolute higher final dose. Accelerated RT implies in the administration of the same number of fractions in a shorter period of time, in other words, an equivalent higher total dose (55).

A pilot study conducted by the Oxford group (56) treated 24 patients with invasive urinary bladder cancer with RT with 54 to 64 Gy, using 1.8 to 2 Gy fractionation, twice a day with a six hour interval between the fractions. Grade 4 or 5 acute urinary toxicity was not registered. Expected survival and local control in two years were $35 \%$ and $56 \%$, respectively. 
Näslund et al. (57) in a randomized study with 168 patients compared two schemes of RT: hyperfractionation with $1 \mathrm{~Gy}$, three times/day and 84 Gy total dose versus 2 Gy, once/day with 64 Gy total dose (conventional fractionation). After a ten year follow-up, a higher local control rate and patient survival was observed in patients submitted to hyperfractionated RT.

A meta-analysis (58) comparing several schemes of hyperfractionated RT used in different kinds of cancer also pointed out a gain in favor of altered fractionation.

A prospective randomized study elaborated at the Royal Marsden Hospital (59) evaluated the effectiveness and the toxicity of accelerated fractionation in the treatment of invasive urinary bladder cancer. Two hundred and twenty nine patients (T2 or T3, N0 or N1, M0) were randomized in two groups: accelerated fractionation - $60.8 \mathrm{~Gy}$ in 32 fractions in 26 days; conventional fractionation - 64 Gy in 32 fractions in 45 days. Acute toxicity was observed in 121 patients of the accelerated fractionation and in 96 patients of the conventional fractionation group. There was no statistically significant difference in local control and disease-free and overall survivals. Accelerated RT was not more effective than the conventional fractionation for T2 and T3 tumors and was associated to higher acute toxicity.

Hypofractionated RT which consists on the administration of larger daily fractions (2.5 Gy to $6 \mathrm{~Gy}$, in general) was also studied. Only one phase III randomized study compared conventional RT (1.5 Gy/day with a total dose of 60 Gy) with hypofractionated RT (3 Gy/day fractions totalizing $30 \mathrm{~Gy}$, followed by a four week rest and a second course with 30 Gy, 1.5 Gy/day). The arm that received hypofractionated RT presented a lower 5-year survival (39\% versus 52\%) (60).

In conclusion, total RT dose is important for locoregional control. Hyperfractioned RT schemes, most probably due to the higher total dose of the treatment, present a real benefit in relation to conventional RT. Accelerated and hypofractionated RT schemes are not recommended, and may present higher toxicity.

\section{Perspectives}

RT current techniques using tridimensional planning systems, intensity modulated RT (IMRT) and image-guidance allow a greater dose-escalation to the treatment targets, with lower doses to the normal adjacent tissues and, consequently, less treatment related toxicity. However, regarding urinary bladder cancer, due to the anatomic alterations inherent to the organ filling that occur inter and intrafractions, the use of high precision techniques may be more limited, both for target definition and protection of organs at risk. Besides that, such techniques are promising and provide the tools for dose-escalation and improvement in local control and, eventually, in survival.

\section{Comprehensive summary}

- RT for superficial tumors is not indicated.

- Pre or postoperative RT is not indicated.

- Radiochemotherapy is an option in the conservative treatment of selected cases of invasive urinary bladder tumors: solitary tumor (T2 to T4) less than $5 \mathrm{~cm}$, absence of hydronephrosis, complete transurethral resection and normal bladder function.

- RT alone should be indicated with palliative intention, or for unresectable tumors, or in inoperable patients, when combination with chemotherapy is not possible.

\section{CONFLICT OF INTEREST}

None declared.

\section{REFERENCES}

1. Jemal A, Bray F, Center MM, Ferlay J, Ward E, Forman D: Global cancer statistics. CA Cancer J Clin. 2011; 61: 69-90. Erratum in: CA Cancer J Clin. 2011;61: 134.

2. American Joint Committee on Cancer. Urinary bladder. In: AJCC Cancer Staging Manual, 7th. New York: SpringerVerlag, 2010; pp. 497-505. 
3. Stein JP, Lieskovsky G, Cote R, Groshen S, Feng AC, Boyd $S$. et al.: Radical cystectomy in the treatment of invasive bladder cancer: long-term results in 1,054 patients. J Clin Oncol. 2001; 19: 666-75.

4. Dall'oglio MF, Nesrallah LJ, Rodrigues P, Nesrallah A, Kauffmann JR, Srougi M: The use of absorbable mechanical suture in orthotopic ileal neobladder replacement. Int Braz J Urol. 2000; 26: 288-92.

5. Castillo OA, Abreu SC, Mariano MB, Tefilli MV, Hoyos J, Pinto I, et al.: Complications in laparoscopic radical cystectomy. The South American experience with 59 cases. Int Braz J Urol. 2006; 32: 300-5.

6. Stenzl A, Sherif H, Kuczyk M: Radical cystectomy with orthotopic neobladder for invasive bladder cancer: a critical analysis of long term oncological, functional and quality of life results. Int Braz J Urol. 2010; 36: 537-47.

7. Beduschi R, Montie JE: Current indications and new possibilities for organ preservation in carcinoma of the bladder. Int Braz J Urol. 2000; 26: 234-41.

8. Gospodarowicz MK, Warde P: The role of radiation therapy in the management of transitional cell carcinoma of the bladder. Hematol Oncol Clin North Am. 1992; 6: 147-68.

9. Quilty PM, Duncan W: Treatment of superficial (T1) tumours of the bladder by radical radiotherapy. Br J Urol. 1986; 58: 147-52.

10. Sawczuk IS, Olsson CA, deVere White R: The limited usefulness of external beam radiotherapy in the control of superficial bladder cancer. Br J Urol. 1988; 61: 330-2.

11. Wolf $\mathrm{H}$, Olsen PR, Højgaard K: Urothelial dysplasia concomitant with bladder tumours: a determinant for future new occurrences in patients treated by full-course radiotherapy. Lancet. 1985; 1: 1005-8.

12. Weiss C, Engehausen DG, Krause FS, Papadopoulos T, Dunst J, Sauer R, et al.: Radiochemotherapy with cisplatin and 5-fluorouracil after transurethral surgery in patients with bladder cancer. Int J Radiat Oncol Biol Phys. 2007; 68: 1072-80.

13. Batata MA, Chu FC, Hilaris BS, Lee MZ, Varesko RW, Lee HS, et al.: Preoperative whole pelvis verus true pelvis irradiation and/or cystectomy for bladder cancer. Int J Radiat Oncol Biol Phys. 1981; 7: 1349-55.

14. Batata MA, Chu FC, Hilaris BS, Kim Y, Lee M, Chang B, et al.: Radiation therapy before cystectomy in the management of patients with bladder cancer. Clin Radiol. 1982; 33: 109-14.

15. Spera JA, Whittington R, Littman P, Solin LJ, Wein AJ: A comparison of preoperative radiotherapy regimens for bladder carcinoma. The University of Pennsylvania experience. Cancer. 1988; 61: 255-62.

16. Parsons JT, Million RR: Planned preoperative irradiation in the management of clinical stage B2-C (T3) bladder carcinoma. Int J Radiat Oncol Biol Phys. 1988; 14: 797-810.

17. Abrahamsen JF, Fosså SD: Long-term morbidity after curative radiotherapy for carcinoma of the bladder. A retrospective study. Strahlenther Onkol. 1990; 166: 580-3.
18. Blackard CE, Byar DP: Results of a clinical trial of surgery and radiation in stages $\mathrm{II}$ and 3 carcinoma of the bladder. $\mathrm{J}$ Urol. 1972; 108: 875-8.

19. Bloom HJ, Hendry WF, Wallace DM, Skeet RG: Treatment of T3 bladder cancer: controlled trial of pre-operative radiotherapy and radical cystectomy versus radical radiotherapy. Br J Urol. 1982; 54: 136-51.

20. Sell A, Jakobsen A, Nerstrøm B, Sørensen BL, Steven K, Barlebo $\mathrm{H}$ : Treatment of advanced bladder cancer category T2 T3 and T4a. A randomized multicenter study of preoperative irradiation and cystectomy versus radical irradiation and early salvage cystectomy for residual tumor. DAVECA protocol 8201. Danish Vesical Cancer Group. Scand J Urol Nephrol Suppl. 1991; 138: 193-201.

21. Miller LS: Bladder cancer: superiority of preoperative irradiation and cystectomy in clinical stages B2 and C. Cancer. 1977; 39(2 Suppl): 973-80.

22. Huncharek M, Muscat J, Geschwind JF: Planned preoperative radiation therapy in muscle invasive bladder cancer; results of a meta-analysis. Anticancer Res. 1998; 18: 1931-4.

23. Dhar NB, Campbell SC, Zippe CD, Derweesh IH, Reuther AM, Fergany A, et al.: Outcomes in patients with urothelial carcinoma of the bladder with limited pelvic lymph node dissection. BJU Int. 2006; 98: 1172-5.

24. Novara G, Svatek RS, Karakiewicz PI, Skinner E, Ficarra $V$, Fradet $Y$, et al.: Soft tissue surgical margin status is a powerful predictor of outcomes after radical cystectomy: a multicenter study of more than 4,400 patients. J Urol. 2010; 183: 2165-70.

25. Duncan W, Quilty PM: The results of a series of 963 patients with transitional cell carcinoma of the urinary bladder primarily treated by radical megavoltage X-ray therapy. Radiother Oncol. 1986; 7: 299-310.

26. Jenkins BJ, Caulfield MJ, Fowler CG, Badenoch DF, Tiptaft $\mathrm{RC}$, Paris AM, et al.: Reappraisal of the role of radical radiotherapy and salvage cystectomy in the treatment of invasive (T2/T3) bladder cancer. Br J Urol. 1988; 62: 343-6.

27. Daehlin L, Haukaas S, Maartmann-Moe H, Medby PC: Survival after radical treatment for transitional cell carcinoma of the bladder. Eur J Surg Oncol. 1999; 25: 66-70.

28. Fossa SD, Aass N, Ous S, Waehre H, Inner K, Hannisdal E: Survival after curative treatment of muscle-invasive bladder cancer. Acta Oncol. 1996; 35(Suppl 8): 59-65.

29. Chung PW, Bristow RG, Milosevic MF, Yi QL, Jewett MA, Warde PR, et al.: Long-term outcome of radiation-based conservation therapy for invasive bladder cancer. Urol Oncol. 2007; 25: 303-9.

30. Shipley WU, Rose MA, Perrone TL, Mannix CM, Heney NM, Prout GR Jr.: Full-dose irradiation for patients with invasive bladder carcinoma: clinical and histological factors prognostic of improved survival. J Urol. 1985; 134: 679-83. 
31. Mameghan H, Fisher R, Mameghan J, Brook S: Analysis of failure following definitive radiotherapy for invasive transitional cell carcinoma of the bladder. Int J Radiat Oncol Biol Phys. 1995; 31: 247-54.

32. Langsenlehner T, Döller C, Quehenberger F, Stranzl-Lawatsch $\mathrm{H}$, Langsenlehner $\mathrm{U}$, Pummer $\mathrm{K}$, et al.: Treatment results of radiation therapy for muscle-invasive bladder cancer. Strahlenther Onkol. 2010; 186: 203-9.

33. Housset M, Maulard C, Chretien Y, Dufour B, Delanian S, Huart J, et al.: Combined radiation and chemotherapy for invasive transitional-cell carcinoma of the bladder: a prospective study. J Clin Oncol. 1993; 11: 2150-7.

34. Coppin CM, Gospodarowicz MK, James K, Tannock IF, Zee $\mathrm{B}$, Carson J, et al.: Improved local control of invasive bladder cancer by concurrent cisplatin and preoperative or definitive radiation. The National Cancer Institute of Canada Clinical Trials Group. J Clin Oncol. 1996; 14: 2901-7.

35. James ND, Hussain SA, Hall E, Jenkins P, Tremlett J, Rawlings $C$, et al.: Results of a phase III randomized trial of synchronous chemoradiotherapy (CRT) compared to radiotherapy $(\mathrm{RT})$ alone in muscle-invasive bladder cancer (MIBC) (BC2001 CRUK/01/004). J Clin Oncol. 2010; 28(Suppl 15): Abstract \# 4517.

36. Rödel C, Grabenbauer GG, Kühn R, Papadopoulos T, Dunst $\mathrm{J}$, Meyer M, et al.: Combined-modality treatment and selective organ preservation in invasive bladder cancer: longterm results. J Clin Oncol. 2002; 20: 3061-71.

37. Manoharan M, Soloway MS: Optimal management of the T1G3 bladder cancer. Urol Clin North Am. 2005; 32: 133-45.

38. Soloway MS, Sofer M, Vaidya A: Contemporary management of stage $\mathrm{T} 1$ transitional cell carcinoma of the bladder. J Urol. 2002; 167: 1573-83.

39. Splinter T, Denis L: Restaging procedures, criteria of response, and relationship between pathological response and survival. Semin Oncol. 1990; 17: 606-12.

40. Shahin 0, Thalmann GN, Rentsch C, Mazzucchelli L, Studer UE: A retrospective analysis of 153 patients treated with or without intravesical bacillus Calmette-Guerin for primary stage $\mathrm{T} 1$ grade 3 bladder cancer: recurrence, progression and survival. J Urol. 2003; 169: 96-100; discussion 100.

41. Peyromaure M, Zerbib M: T1G3 transitional cell carcinoma of the bladder: recurrence, progression and survival. BJU Int. 2004; 93: 60-3.

42. May M, Helke C, Nitzke T, Vogler H, Hoschke B: Survival rates after radical cystectomy according to tumor stage of bladder carcinoma at first presentation. Urol Int. 2004; 72: 103-11.

43. Caffo 0, Fellin G, Graffer U, Mussari S, Tomio L, Galligioni $\mathrm{E}$ : Gemcitabine and radiotherapy plus cisplatin after transurethral resection as conservative treatment for infiltrating bladder cancer: Long-term cumulative results of 2 prospective single-institution studies. Cancer. 2011; 117: 1190-6.
44. Kotwal S, Munro N: Radiotherapy in localized bladder cancer: what is the evidence? Curr Opin Urol. 2010; 20: 426-31.

45. Kachnic LA, Kaufman DS, Heney NM, Althausen AF, Griffin PP, Zietman AL, et al.: Bladder preservation by combined modality therapy for invasive bladder cancer. J Clin Oncol. 1997; 15: 1022-9.

46. Shipley WU, Kaufman DS, Heney NM, Althausen AF, Zietman AL: An update of combined modality therapy for patients with muscle invading bladder cancer using selective bladder preservation or cystectomy. J Urol. 1999; 162: 445-50; discussion 450-1.

47. Tester W, Porter A, Asbell S, Coughlin C, Heaney J, Krall $J$, et al.: Combined modality program with possible organ preservation for invasive bladder carcinoma: results of RTOG protocol 85-12. Int J Radiat Oncol Biol Phys. 1993; 25: 783-90.

48. Arias F, Domínguez MA, Martínez E, Illarramendi JJ, Miquelez S, Pascual I, et al.: Chemoradiotherapy for muscle invading bladder carcinoma. Final report of a single institutional organ-sparing program. Int J Radiat Oncol Biol Phys. 2000; 47: 373-8.

49. Shipley WU, Winter KA, Kaufman DS, Lee WR, Heney NM, Tester WR, et al.: Phase III trial of neoadjuvant chemotherapy in patients with invasive bladder cancer treated with selective bladder preservation by combined radiation therapy and chemotherapy: initial results of Radiation Therapy Oncology Group 89-03. J Clin Oncol. 1998; 16: 3576-83.

50. Perdonà $\mathrm{S}$, Autorino R, Damiano R, De Sio M, Morrica $\mathrm{B}$, Gallo $\mathrm{L}$, et al.: Bladder-sparing, combined-modality approach for muscle-invasive bladder cancer: a multi-institutional, long-term experience. Cancer. 2008; 112: 75-83.

51. Neoadjuvant cisplatin, methotrexate, and vinblastine chemotherapy for muscle-invasive bladder cancer: a randomised controlled trial. International collaboration of trialists. Lancet. 1999; 354: 533-40. Erratum in: Lancet. 1999; 354: 1650.

52. International Collaboration of Trialists; Medical Research Council Advanced Bladder Cancer Working Party (now the National Cancer Research Institute Bladder Cancer Clinical Studies Group); European Organisation for Research and Treatment of Cancer Genito-Urinary Tract Cancer Group; Australian Bladder Cancer Study Group; National Cancer Institute of Canada Clinical Trials Group; Finnbladder et al.: International phase III trial assessing neoadjuvant cisplatin, methotrexate, and vinblastine chemotherapy for muscle-invasive bladder cancer: long-term results of the BA06 30894 trial. J Clin Oncol. 2011; 29: 2171-7.

53. Moonen L, vd Voet $H$, de Nijs R, Horenblas S, Hart AA, Bartelink $\mathrm{H}$ : Muscle-invasive bladder cancer treated with external beam radiation: influence of total dose, overall treatment time, and treatment interruption on local control. Int J Radiat Oncol Biol Phys. 1998; 42: 525-30. 
54. Pos FJ, Hart G, Schneider C, Sminia P: Radical radiotherapy for invasive bladder cancer: What dose and fractionation schedule to choose? Int J Radiat Oncol Biol Phys. 2006; 64: 1168-73.

55. Kob D, Arndt J, Kriester A, Schwenk M, Kloetzer KH: Results of percutaneous radiotherapy of bladder cancer using 1 and 2 series of irradiation. Strahlentherapie. 1985; 161: 673-7.

56. Cole DJ, Durrant KR, Roberts JT, Dawes PJ, Yosef $H$, Hopewell JW: A pilot study of accelerated fractionation in the radiotherapy of invasive carcinoma of the bladder. $\mathrm{Br} \mathrm{J}$ Radiol. 1992; 65: 792-8.

57. Näslund I, Nilsson B, Littbrand B: Hyperfractionated radiotherapy of bladder cancer. A ten-year follow-up of a randomized clinical trial. Acta Oncol. 1994; 33: 397-402.

\section{EDITORIAL COMMENT}

The authors reviewed the current status of radiation therapy (RT) in the management of bladder cancer, which become more attractive to the patients due to the possibility to retain the urinary bladder and having better quality of life. Beside that, this approach will be an alternative for patients who could not underwent operation due to their medical condition. Based on the recent studies the authors concluded that radiochemotherapy is an alternative for selected muscle invasive bladder cancer patients instead of radical operation.

Several studies reported that trimodality treatment: complete TUR-BT, chemotherapy, and RT could have similar results compared to radical cystectomy. Five-year overall survival reaching $70 \%$ and about $80 \%$ of the surviving patients could preserve their bladder (Ref. 12, 36, and 43 in the article). The limitations are delivery of ad-
58. Stuschke M, Thames HD: Hyperfractionated radiotherapy of human tumors: overview of the randomized clinical trials. Int J Radiat Oncol Biol Phys. 1997; 37: 259-67.

59. Horwich A, Dearnaley D, Huddart R, Graham J, Bessell E, Mason $\mathrm{M}$, et al.: A randomised trial of accelerated radiotherapy for localised invasive bladder cancer. Radiother Oncol. 2005; 75: 34-43.

60. Kob D, Arndt J, Kriester A, Schwenk M, Kloetzer KH: Results of percutaneous radiotherapy of bladder cancer using 1 and 2 series of irradiation. Strahlentherapie. 1985; 161: 673-7.

Correspondence address: Dr. Gustavo Nader Marta Department of Radiation Oncology - Oncology Center Hospital Sirio-Libanes Rua Dona Adma Jafet, 91 Sao Paulo - SP, 01308-050, Brazil Telephone: +55 11 3155-0558 E-mail: gnmarta@uol.com.br

equate dose of chemotherapeutic agents into the tumor site and its systemic adverse events. To overcome this problem, some investigators developed novel approach such as balloon-occluded intra-arterial infusion of gemcitabine and cisplatin with concomitant hemodialysis and concurrent RT (1). In the same time, more understanding of the tumor biology and incorporating molecular markers as predictive factor for successful treatment lead toward better patient selection; development of new chemotherapeutic, including targeted therapy drugs, in combination with RT, will improve the effectiveness of this treatment strategy for tumor control (2).

Important thing to be remembered when choosing this treatment modality is the need for strict follow-up by periodic cystoscopy and also the patients should be aware of the possibility for cystectomy that needs to be done in case of disease progression. 


\section{REFERENCES}

1. Azuma H, Inamoto T, Takahara K, Ibuki N, Nomi H, Yamamoto K, et al.: Neoadjuvant and adjuvant chemotherapy for locally advanced bladder carcinoma: development of novel bladder preservation approach, Osaka Medical College regimen. Int J Urol. 2012; 19: 26-38.
2. Balar A, Bajorin DF, Milowsky MI: Management of invasive bladder cancer in patients who are not candidates for or decline cystectomy. Ther Adv Urol. 2011; 3: 107-17.

Dr. Rainy Umbas University of Indonesia Faculty of Medicine, Division of Urology Jakarta, Indonesia E-mail: rainy.umbas@gmail.com 